\title{
Thermo-physical Behavior of Nanomaterials with the Change in Size and Shape
}

\author{
Ratan Lal Jaiswal ${ }^{1}$, Brijesh Kumar Pandey ${ }^{2 *}$, Dishani Mishra ${ }^{2}$, and Huda Fatma ${ }^{2}$ \\ ${ }^{1}$ Department of Physics, Government Degree College, Dhadha Bujurg, Hata, Kushinagar UP (India) \\ ${ }^{2}$ Department of Physics and Material Science, MMM University of Technology, Gorakhpur UP (India) \\ ${ }^{2}$ Corresponding Author: email- bkpmmmec11@gmail.com
}

\begin{abstract}
Thermo elastic properties of nanomaterials has been very interesting among the researchers during the last decade, still it is a great challenge to predict the exact thermoelastic behaviour of nanomaterials. In the present work we have studied the volume thermal expansion coefficient of low dimensional solid and the variation of Young's modulus with change in temperature for different shapes of nanomaterials by considering the effect of packing factor. We have computed the volume thermal expansion coefficient of Silver $(\mathrm{Ag})$, Aluminum $(\mathrm{Al})$, Copper $(\mathrm{Cu})$, and Lead $(\mathrm{Pb})$ with their varying size. The effect of temperature on Young's modulus of Silver (Ag), Gold (Au), Nickel (Ni), Copper (Cu) and Silicon $(\mathrm{Si})$ has also been studied. The computed results are compared with available experimental data which confirms that the volume thermal expansion coefficient increases with reduction in size of the nanomaterials. It has been also observed that the Young's modulus has linear decrement with increase in temperature which indicates that Young's modulus of nanomaterials has negative temperature coefficient.
\end{abstract}

Received 28 January 2021, Revised 12 February 2021, Accepted 14 February 2021

\section{Keywords: Thermal expansion coefficient; packing factor; elastic properties; nanostructures.}

\section{Introduction}

Nanomaterials are characterized as materials which have at least one of the dimensions measuring less than $100 \mathrm{~nm}$ are known as nanomaterials. Nowadays nanomaterials have become one of the most vigorous fields of research because at this scale a unique optical, thermodynamic, magnetic, electrical and other property emerges. Because of their small size, nanomaterials have distinct properties when compared to the bulk type of same material. This change is profoundly because of the quantum confinement effect and larger surface area. At the point where the size of particle reduces at nanolevel, the surface to volume ratio increases quickly. Many investigations of size and temperature dependent thermal and mechanical property of the nanoscaled materials are being carried out for many years. In this study, our aim is to examine the effect of size on volume thermal expansion and effect of temperature on Young's modulus of nanomaterials. Different group of researchers did the both experimental and theoretical works on these properties [1-9]. Out of the different theoretical models, the most effective model for thermal expansivity was given by R. Kumar and M. Kumar [10] based upon the most basic concept of cohesive energy of the materials. As the material's dimensions approaches from bulk to the nanoscale, the thermal expansion coefficient changes with change in size of the material. This fact is experimentally observed many times. Change in size or volume of a given material of fixed mass in retaliation to change in temperature is termed as thermal expansion. Upon heating a substance, its kinetic energy increases and thus molecules begin to vibrate more and usually keep up greater mean separation. The change in size of an object with change in temperature is suitably explained by the thermal expansion coefficient. It measures the change in size for unit degree rise in temperature at a constant pressure.

The temperature-dependent mechanical properties such as elastic property of nanomaterials is significant to abstain to processing defects, to understand and to enhance the hightemperature performance. The stability of devices at nanolevel is related to elasticity and its temperature dependence. For instance, the elastic instability by increasing the temperature may cause the failure of integrated circuits as well as other microelectronic devices. Thus the study of the effect of temperature on elastic modulus is more useful.

The stability or consistency of nanoscale devices is related to elastic properties of nanomaterials and temperature, thus the analysis of the elastic properties of nanomaterials under temperature is very important. On the basis of above discussions we can conclude that the elastic modulus of nanomaterials vary not only with the temperature but also make significant impact on the volume thermal expansivity of the material. It has been found that, as temperature increases the effect on phonon energy and strain may increases and decreases, respectively. Our special attention was focused to investigate the effect of temperature on elastic modulus uniquely Young's modulus of nanomaterials at different thickness. For theoretical demonstration, we consider a well-established model proposed by Sharma et.al. and considered a very simple and fundamental relation between thermal behavior and elastic behavior of nanomaterials [11].

\section{Theoretical Analysis}

Thermal and elastic behavior of nanomaterials is directly related to the cohesive energy of the nanomaterials. It is also reported that the thermal expansivity of the nanomaterial is closely related to its cohesive energy. Total cohesive energy of the nanosolid $\mathrm{E}_{\text {total }}$ is given in terms of the cohesive energy of its surface and inner atoms, which can be given as [12]-

$E_{\text {total }}=E_{o}(n-N)+\frac{N}{2} E_{o}$

where, $E_{o}$ is the cohesive energy of an atom, $n$ is the total number of atoms of the nanosolid, $\mathrm{N}$ is the total number of 
surface atoms. If $\mathrm{E}_{\mathrm{N}}$ is the cohesive energy per mole of the nanosolid given by

$$
E_{N}=\frac{A E_{\text {total }}}{n}
$$

where, $\mathrm{A}$ is the Avogadro number and $\mathrm{E}_{\mathrm{B}}$ is the cohesive energy per mole of the equivalent bulk sample given by $\mathrm{E}_{\mathrm{B}}=$ $\mathrm{A} \mathrm{E}_{\mathrm{o}}$, then Eq. (1) becomes:

$$
E_{N}=E_{B}\left(1-\frac{N}{2 n}\right)
$$

The cohesive energy of nanomaterials is directly dependent to its elastic modulus of elasticity [10], thus we have the following relation:

$$
Y_{N}=Y_{B}\left(1-\frac{N}{2 n}\right)
$$

where $Y_{N}$ is the Young's modulus of elasticity of the nanosolid and $Y_{B}$ is the Young's modulus of elasticity of the corresponding bulk material.

Kumar et. al. uses the relation between bulk modulus (B) and thermal expansion coefficient $(\alpha)$ i.e. $\alpha B=$ constant, to formulate the expression for the variation of volume thermal expansion coefficient with the size of the nanoparticles [10]. According to this relation

$\alpha_{N}=\alpha_{B}\left(1-\frac{N}{2 n}\right)^{-1}$

\subsection{Volume Thermal Expansion Coefficient of Nanoparticle}

If we consider the case of spherical nanocrystal of diameter $\mathrm{D}$ with atomic diameter $\mathrm{d}$, then the total number of atoms $\mathrm{n}$ is given by [13]

$$
n=\frac{D^{3}}{d^{3}} \eta
$$

where $\eta$ is the atomic packing factor which may be defined as the fraction of volume of crystal occupied by its constituent atoms.

$$
\eta=\frac{V_{\text {atom }}}{V_{\text {cell }}}
$$

where, $\mathrm{V}_{\text {atom }}$ is the volume of an atom and $\mathrm{V}_{\text {cell }}$ is the volume of the unit cell. Now, the surface area of nanoparticle is $\pi \mathrm{D}^{2}$ and area of each surface atom that contributes to the surface area of nanoparticle is $\pi \mathrm{d}^{2} / 4$.

Thus, the total surface atoms $\mathrm{N}$ is given by [14]

$N=\frac{4 D^{2}}{d^{2}}$

Therefore, using Eq. (6) and Eq. (7) we have

$$
E_{N}=E_{B}\left(1-\frac{2 d}{\eta D}\right)
$$

Hence,

$$
\alpha_{N}=\alpha_{B}\left(1-\frac{2 d}{\eta D}\right)^{-1}
$$

2.2 Volume Thermal Expansion Coefficient of Nanowire

The volume of a nanowire of diameter $\mathrm{L}$ and height $\mathrm{h}$, is $\pi \mathrm{L}^{2} h / 4$, thus in reference of Eq. (6), total number of atoms $n$ becomes $n=\frac{3 L^{2} h}{2 d^{3}} \eta$

The total surface area of the nanowire will be $\left(\pi \mathrm{L}^{2} / 2\right)+\pi \mathrm{Lh}$. Number of atoms on the surface $\mathrm{N}$ is

$N=\frac{2 L^{2}+4 L h}{d^{2}}$

Hence, from Eq. (10) and Eq. (11) we have

$\frac{N}{2 n}=\frac{2 d}{3 \eta}\left(\frac{2}{L}+\frac{1}{h}\right)$

For nanowire, $\mathrm{h}>>\mathrm{L}$, thus we have from above equation

$\frac{N}{2 n}=\frac{4 d}{3 L \eta}$

Hence Eq. (9) becomes

$\alpha_{N}=\alpha_{B}\left(1-\frac{4 d}{3 L \eta}\right)^{-1}$

\subsection{Volume Thermal Expansion Coefficient of Nanofilm}

If we consider Eq. (12) with similar meaning of the parameters in the case of nanofilms, then $\mathrm{L}>\mathrm{h}$, so from Eq. (12)

$\frac{N}{2 n}=\frac{2 d}{3 h \eta}$

Thus,

$\alpha_{N}=\alpha_{B}\left(1-\frac{2 d}{3 h \eta}\right)^{-1}$

Eqs. (9), (13) and (14) give the relation between volume thermal expansion coefficient of the nanomaterials $\left(\alpha_{N}\right)$ and the bulk material $\left(\alpha_{\mathrm{B}}\right)$, depending on atomic diameter $(\mathrm{d})$ and the size of the nanomaterials.

Kumar et. al. has proposed a model for studying the temperature dependence of thermal expansion of nanomaterials [15], according to this model

$\frac{\alpha_{N}}{\alpha_{B}}=\left[1-\alpha_{N} \delta_{T}\left(T-T_{0}\right)\right]^{-1}$

Here, $\alpha_{N}$ and $\alpha_{B}$ is volume thermal expansion of nanomaterials and corresponding bulk materials, respectively. $\delta_{\mathrm{T}}$ is known as the Anderson-Gruneisen parameter. Putting the value $\alpha_{N}$ in Eq. (15) from the Eq. (5) we get,

$\frac{\alpha_{N}}{\alpha_{B}}=\left[1-\alpha_{B}\left(1-\frac{N}{2 n}\right)^{-1} \delta_{T}\left(T-T_{0}\right)\right]^{-1}$

For nanomaterials thermal and elastic behavior are inverse to each other [11]. Accordingly, we can compose a relation to study the temperature dependence on elastic properties of nanomaterials.

Therefore, finally we can write an expression for the temperature dependence of Young's modulus for nanomaterials, which is given as:

$\frac{Y_{N}}{Y_{B}}=\left[1-\alpha_{B}\left(1-\frac{N}{2 n}\right)^{-1} \delta_{T}\left(T-T_{0}\right)\right]$

Here, $Y_{N}$ is Young's modulus of nanomaterials. $Y_{B}$ is young modulus corresponding to bulk materials. 
The fraction $\frac{N}{n}$ represents the number of surface atom in comparison to the total number of atoms in the material which depends on the shape of the nanomaterials. The values of this fraction for different shapes of nanomaterials are calculated in previous section.

\section{For spherical nanosolid}

$$
\frac{Y_{N}}{Y_{B}}=\left[1-\alpha_{B}\left(1-\frac{2 d}{\eta D}\right)^{-1} \delta_{T}\left(T-T_{0}\right)\right]
$$

\section{For nanowire}

$$
\frac{Y_{N}}{Y_{B}}=\left[1-\alpha_{B}\left(1-\frac{4 d}{3 \eta L}\right)^{-1} \delta_{T}\left(T-T_{0}\right)\right]
$$

\section{For nanofilm}

$$
\frac{Y_{N}}{Y_{B}}=\left[1-\alpha_{B}\left(1-\frac{2 d}{3 \eta h}\right)^{-1} \delta_{T}\left(T-T_{0}\right)\right]
$$

Eqs. (18)-(20) are the general relations to examine the impact of temperature on Young's modulus of all three dimensions viz. nanosolid, nanowires and nanofilms, respectively.

\section{Results}

For the better understanding of the above theory, we have studied the variation of the thermal expansion coefficient with size of the nanostructured materials and the variation of the Young's modulus of different nanomaterials with temperature for their fixed sizes.

In the first part of our work we have used Eqs. (9), (13) and (14) for computing the size dependent volume thermal expansion coefficient of all the three dimensions for $\mathrm{Al}$ (aluminium), $\mathrm{Ag}$ (silver), $\mathrm{Cu}$ (copper), and $\mathrm{Pb}$ (lead) nanosolids. Input data for computing the results are obtained from the Table 1. Computed results are plotted in the Figures $1-4$, and the obtained results are also compared with the available experimental results.

Table 1. Required data for the atomic diameter $d(\mathrm{~nm})$, atomic packing fraction $\eta$ and the bulk volume thermal

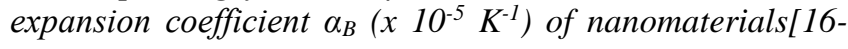

\begin{tabular}{llll}
\hline Nanomaterial & $\begin{array}{l}\text { Atomic } \\
\text { diameter d } \\
(\mathrm{nm})\end{array}$ & $\begin{array}{l}\text { Atomic } \\
\text { Packing } \\
\text { Fraction } \eta\end{array}$ & $\begin{array}{l}\alpha_{\mathrm{B}} \\
\left(\mathrm{x} 10^{-5} \mathrm{~K}^{-1}\right)\end{array}$ \\
\hline $\mathrm{Al}$ & 0.2860 & 0.74 & 6.9 \\
$\mathrm{Ag}$ & 0.2880 & 0.74 & 5.83 \\
$\mathrm{Cu}$ & 0.2560 & 0.74 & 4.9 \\
$\mathrm{~Pb}$ & 0.3898 & 0.74 & 8.7 \\
\hline 21$]$ & & &
\end{tabular}

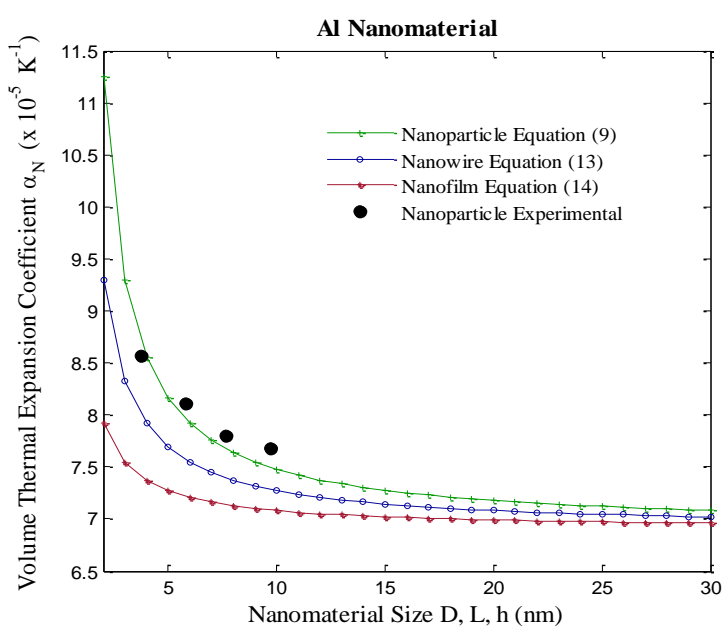

Figure 1. Variation of volume thermal expansion coefficient of Aluminium nanoparticle, nanowire and nanofilm with change in size $D, L$ and $h(n m)$ respectively. The experimental points represent nanoparticles [17].

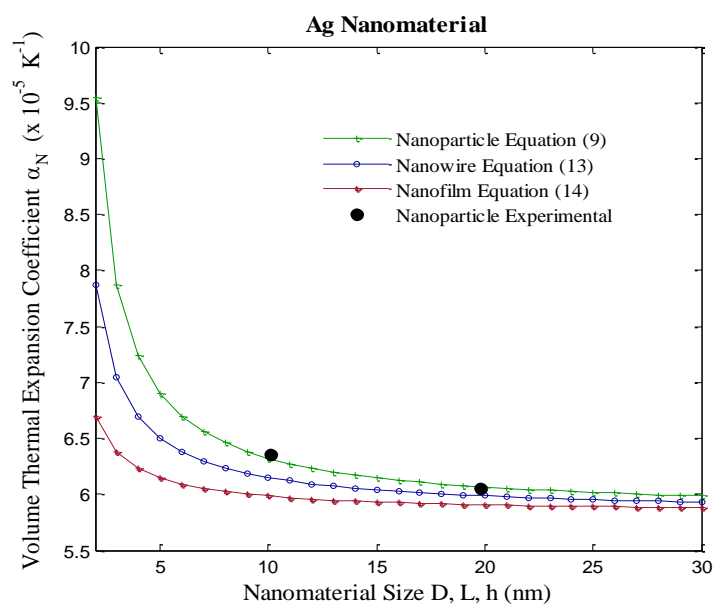

Figure 2. Variation of volume thermal expansion coefficient of silver nanoparticle, nanowire and nanofilm with change in size $D, L$ and $h(\mathrm{~nm})$ respectively. The experimental points represent nanoparticles. [17].

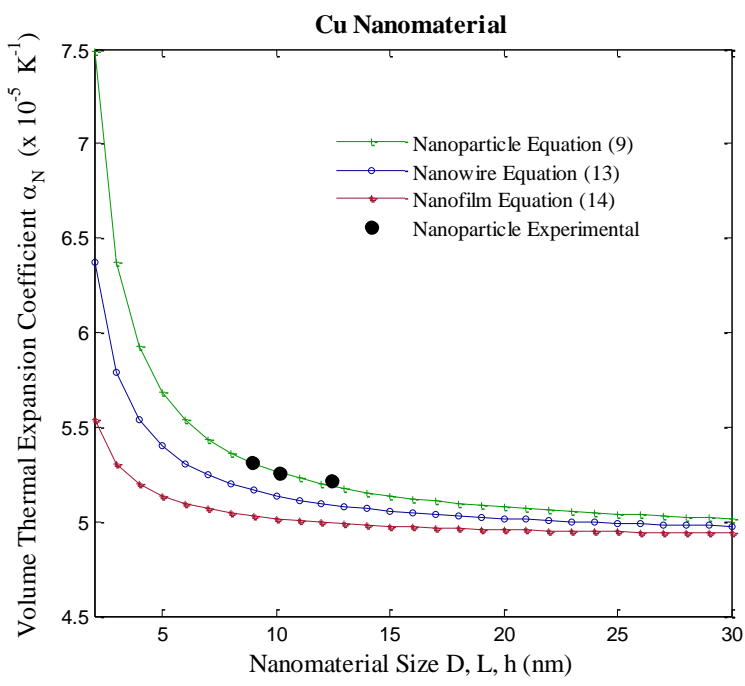

Figure 3. Variation of volume thermal expansion coefficient of copper nanoparticle, nanowire and nanofilm with change in size $D, L$ and $h(\mathrm{~nm})$ respectively. The experimental points represent nanoparticles [17]. 


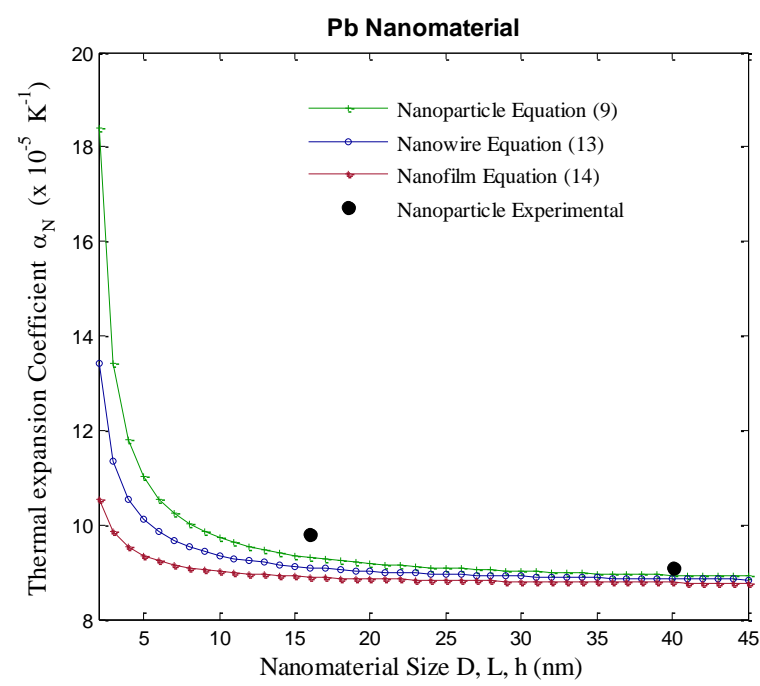

Figure 4. Variation of volume thermal expansion coefficient of lead nanoparticle, nanowire and nanofilm with change in size $D, L$ and $h(n m)$ respectively, the experimental points represent nanoparticles [22].

Fig.1-4 are showing the variation of volume thermal expansion coefficient of nanoparticles, nanowire and nanofilms with their sizes. The available experimental results are also compared with our computed results. Our computed results can be discussed as following:

(a) The size dependent volume thermal expansion coefficient of $\mathrm{Al}, \mathrm{Ag} \mathrm{Cu}, \mathrm{Pb}$ nanoparticles is verified with the available experimental results. From the figures 1, 2, 3 and 4 , it is well observed that our theoretical results are in good agreement with the available experimental data. This validates the model for thermal expansivity suggested in the present work.

(b) The nanomaterials show an increasing trend of volume thermal expansion coefficient with decrease in its size. It is marked here that the variation of thermal expansion coefficient is effective in lower nanoscale, above which its value slowly approaches towards the bulk value of the material. Available experimental results and predicted curves shows same trend of variation.

Another fact that is noticeable from above figures is that at a particular size of the nanomaterial, the thermal expansion coefficient of the nanoparticles is higher than that of the nanowire and the least in case of nanofilms. The reason to this fact lies in the ratio N/2n; it can also be explained by cohesive energy of nanomaterials. It is observed that there are a greater number of atoms are revealed to the surface than in the interior in case of nanoparticles in comparison to the other two dimensions. Similarly, the least number of atoms are exposed to the surface than in the interior in nanofilms. Since larger the number of surface to interior atoms, lesser is the cohesive energy required for that material. Thus, the cohesive energy required for the material of a particular size is highest for nanofilms and the least for nanoparticles. Since the cohesive energy is inversely proportional to thermal expansivity thus, the volume thermal expansion coefficient for nanofilms is lowest than nanowires and nanoparticles of at the same size.
In the second part of our work, we have used Eqs. (18)(20) for computing the variation of Young's modulus with temperature for $\mathrm{Au}$ (gold), $\mathrm{Ag}$ (silver), $\mathrm{Ni}$ (nickel), $\mathrm{Cu}$ (copper), Si (silicon) nanosolids at particular size. In our present work, we have focused to predict the effect of temperature on Young's modulus of FCC structured nanofilms. Input data to computing the results are obtained from the Table 2. Computed results are shown in the Figures 5-11, which has also been compared with the available experimental results.

Table 2. Required data for the atomic diameter $d(\mathrm{~nm})$, bulk volume thermal expansion coefficient $\alpha_{b}\left(10^{-6} \mathrm{~K}^{-1}\right)$ and bulk Young's modulus $Y_{b}(G P a)$

\begin{tabular}{llll}
\hline Nanomaterials & $\begin{array}{l}\text { Atomic } \\
\text { diameter d } \\
(\mathrm{nm})\end{array}$ & $\begin{array}{l}\text { Bulk volume } \\
\text { thermal } \\
\text { expansion } \alpha_{\mathrm{b}} \\
\left(10^{-6} \mathrm{~K}^{-1}\right)\end{array}$ & $\begin{array}{l}\text { Bulk } \\
\text { Young's } \\
\text { modulus } \mathrm{Y}_{\mathrm{b}} \\
(\mathrm{GPa})\end{array}$ \\
\hline $\mathrm{Au}$ & 0.2880 & 14.2 & 78 \\
$\mathrm{Ag}$ & 0.3300 & 18.9 & 83 \\
$\mathrm{Ni}$ & 0.2480 & 13.4 & 200 \\
$\mathrm{Cu}$ & 0.2556 & 16.5 & 130 \\
$\mathrm{Si}$ & 0.3368 & 4.2 & 47 \\
\hline
\end{tabular}

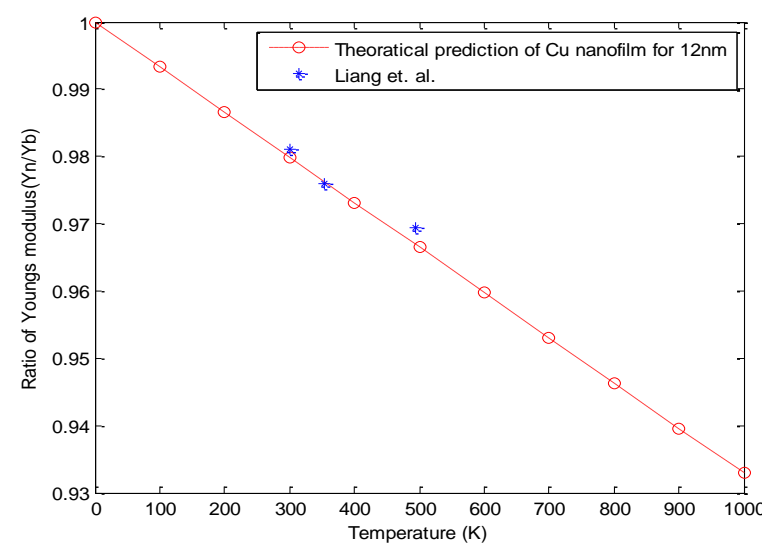

Figure 5. Variation in Young's modulus of Copper (Cu) nanofilm with temperature at $12 \mathrm{~nm}$ thickness. The simulated data is shown by * for Cu nanofilm [23].

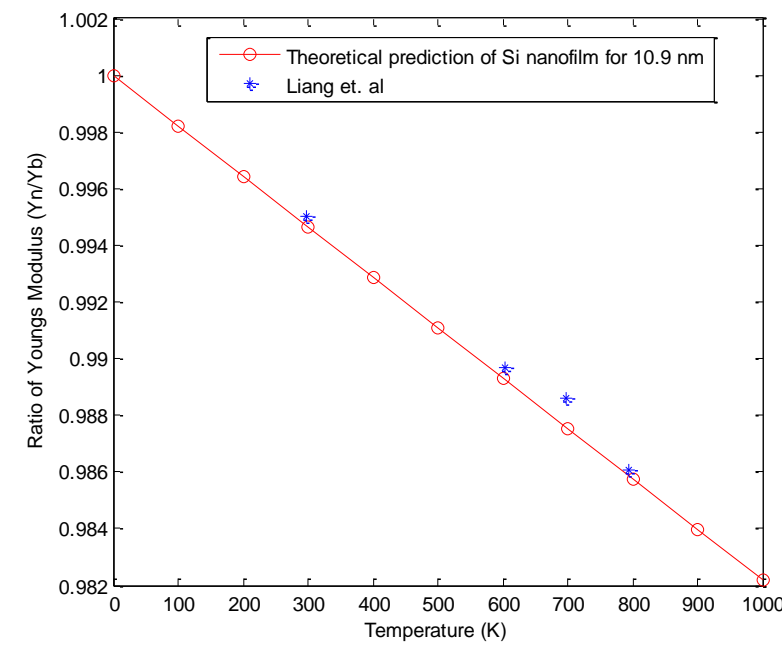

Figure 6. The deviation in Young's modulus ratio of Silicon (Si) nanofilm with temperature at 10.9nm thickness. The simulated data is shown by * for Si nanofilm [23]. 


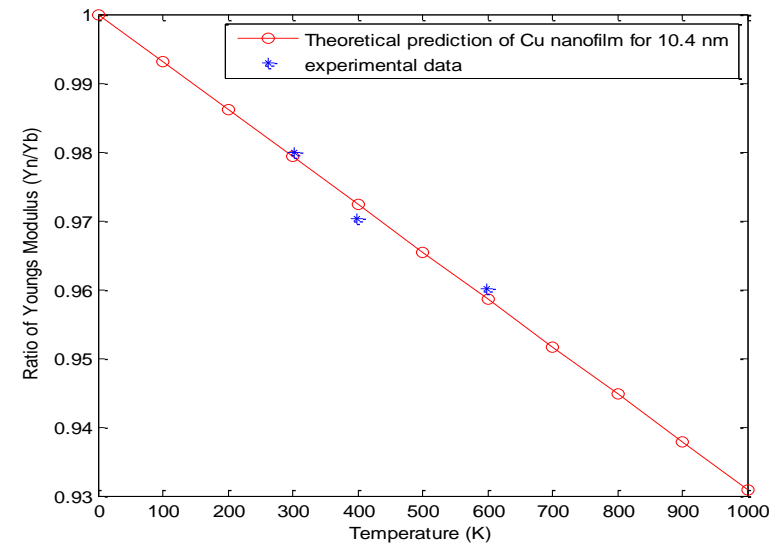

Figure 7. The deviation in Young's modulus ratio of copper (Cu) nanofilm with temperature at 10.4nm thickness. The symbol * represents experimental data [24].

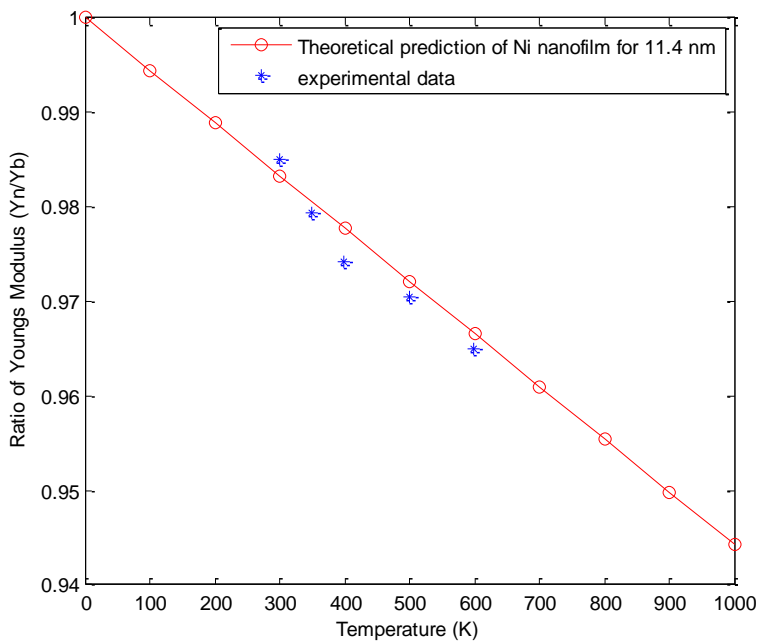

Figure 8. The deviation in Young's modulus ratio of Nickel (Ni) nanofilm with temperature at 11.4nm thickness. The symbol * represents experimental data available [24].

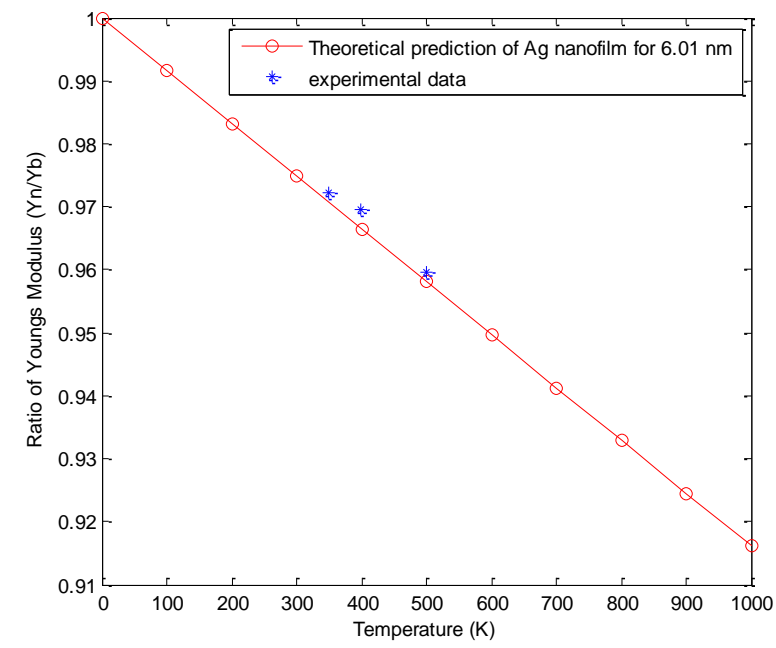

Figure 9. The variation of Young's modulus of Silver (Ag) nanofilm with temperature at $6.01 \mathrm{~nm}$ thickness. The symbol * represents experimental data available [24].

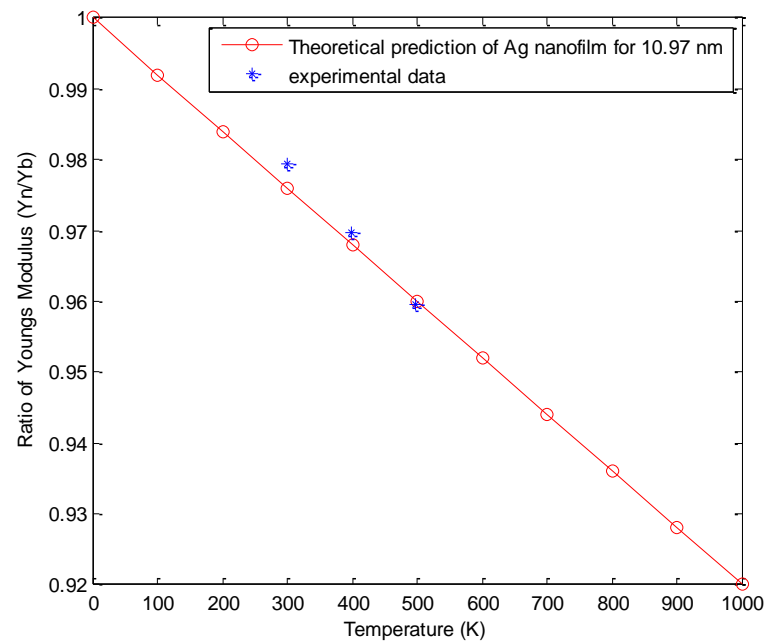

Figure 10. The deviation in Young's modulus ratio of Silver (Ag) nanofilm with temperature at 10.97nm thickness. The symbol * represents experimental data available [24].

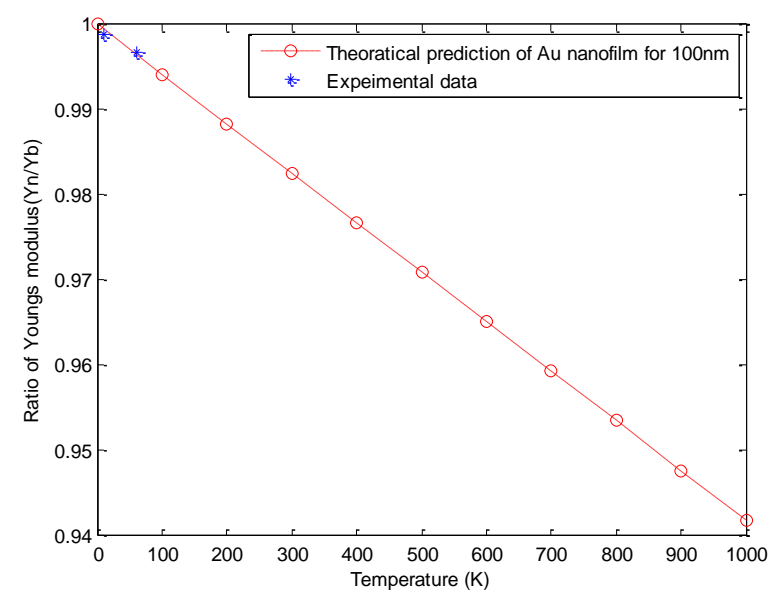

Figure 11. The deviation in Young's modulus ratio of gold (Au) nanofilm with temperature at 100nm thickness. The symbol * represents experimental data available [9].

Sharma et. al. [11] predicted the model for studying the temperature effect on Young's modulus of $\mathrm{Cu}$ nanofilm at $10.4 \mathrm{~nm}$ and $12 \mathrm{~nm}$ and $\mathrm{Si}$ nanofilm at $10.9 \mathrm{~nm}$ thickness where they compared their work with the experimental data. It has been found that the model agrees well with experimental available data. So, in this work, we further implement the model for Au nanofilm at $100 \mathrm{~nm}$, Ni nanofilm at $11.4 \mathrm{~nm}, \mathrm{Ag}$ nanofilm at $6.01 \mathrm{~nm}$ and $10.97 \mathrm{~nm}$ thickness. The variation of Young's modulus for varying sizes of $\mathrm{Cu}$ and $\mathrm{Ag}$ have been shown in Figures 5 and 7; Figures 9-10. From these graphs it is clear that the Young's modulus decreases with decrease in size in each cases [5]. Since the experimental data on temperature dependence of Young's modulus for different shapes (viz. nanowires and nanosolids) are not available in literature. Therefore, we predict the results in the absence of experimental data.

We have verified our results using the experimental data available for $\mathrm{Ag}, \mathrm{Cu}, \mathrm{Ni}$ and $\mathrm{Au}$ nanoparticles. Our computed results for $\mathrm{Cu}$, and $\mathrm{Si}$ are also compared with the simulated results reported by other researchers [25]. There is a very good agreement between our computed results and the experimental results or the simulated results reported by [25]. Variation of Young's modulus with temperature for 
different nanomaterials are plotted in the Figures 5-11. The computed values for Young's modulus along with available experimental data reported by Liang et.al. [23] is shown Figures 5 and 6 . This section includes the discussion on the facts which are marked in the plots showing the variation of Young's modulus with temperature as described below:

(a) Validation of our results is based on the available experimental results and the simulated results reported by Liang et. al [23]. They performed molecular dynamics simulations of the biaxial tension of copper nanofilms at various temperature to validate the temperature impact on the elastic modulus. In the present work the temperature dependence of Young's modulus obtained from Eq. (20) are reported in Figures 7-11 along with the corresponding experimental data. It is obvious from the graphs that, the predicted results showing the temperature impact on the Young's modulus has excellent agreement with experimental values for nanomaterials. Using same theory Eqs. (18)-(20) have been used for predicting the variation in Young's modulus with temperature for other nanomaterials.

(b) It is observed that the Young's modulus decreases with the increase in the temperature due to the increase in the amplitude of atomic vibrations and thermal expansion. At a point when temperature is higher, the atomic thermal vibration increases which will lead for the changes in lattice potential energy. Thus, the Young's modulus changes accordingly. As temperature increases the Young's modulus of nanofilms decreases linearly up to $1000^{\circ} \mathrm{K}$, and perhaps well beyond. Hence, it is noticeable that the Young's modulus of nanomaterials has negative temperature coefficients.

\section{Conclusion}

The behavior of volume thermal expansion coefficient with size and the Young's modulus of nanomaterials with the change in temperature have been studied. It is concluded that, the volume thermal expansion coefficient increases with reduction in size of the nanomaterial (for all the three dimensions) and at a particular size of the material, the thermal expansion coefficient of nanoparticles is higher than that of nanowires and the least for nanofilms.

It is also observed that the Young's modulus decreases with increase in temperature linearly up to $1000^{\circ} \mathrm{K}$ and perhaps well beyond. The negative temperature coefficients of Young's modulus of nanomaterials has also been observed. From the variation of Young's modulus for varying sizes of $\mathrm{Cu}$ and $\mathrm{Ag}$, it is clear that the Young's modulus decreases with decrease in size. In our work much weightage has been given to packing fraction of crystals, because due to this key factor our predictions are much closer to the experimental evidences than the earlier reported simulated results.

\section{References}

[1] M. Singh, "Modelling of thermal expansion coefficient and specific heat of nanomaterials", National university of Lesotho, International Science, Technology and Innovation Conference and Expo, pp. 81-84, 2018.

[2] M. Goyal and B. R. K. Gupta, "Shape, size and temperature dependency of thermal expansion, lattice parameter and bulk modulus in nanomaterials", Journal of Physics, 90(80), pp. 1-8, 2018.
[3] M. Goyal and B. R. K. Gupta, "Study of Shape, Size and Temperature-Dependent Elastic Properties of Nanomaterials", Modern Physics Letters B, vol. 33(26), 1950310, 2019.

[4] M. Manua, M. Singh, V. Dubey, "Impact of Size on Moduli of Elasticity of Nanosolid, Nanowire and Nanofilm", American International Journal of Research in Formal, Applied \& Natural Sciences, vol. 21, pp. 3241, 2018.

[5] Brijesh K Pandey and Ratan L Jaiswal, "Prediction of Elastic Moduli of Metallic Nanoparticles", International Journal of Materials Science, vol. 12, pp. 0973-4589, 2017.

[6] Sachin, B. K. Pandey and R. L. Jaiswal, "Modelling for the prediction of melting temperature for metallic nanoparticles", Advanced Science, Engineering and Medicine, Vol. 12(1), 27-30, 2020.

[7] Q. Fu, Z. Cui, Y. Xue and H. Duan, "Research of size and shape dependent thermodynamic properties of the actual melting process of nanoparticles", The Journal of Physical Chemistry, v. 122, pp. 15713-15722, 2018.

[8] H. Chhabra, S. Bhatt and M. Kumar, "Search of a model for melting temperature and cohesive energy of nanomaterials", Indian Journal of Pure and Applied Physics, v. 57, pp. 361-368, 2019.

[9] C. Birleanu, M. Pustan, V. Merie, R. Muller, R. Voicu, A. Baracu and S. Craciun, "Temperature Effect on the Mechanical Properties of Gold Nanofilms with Different Thickness", Materials Science and Engineering (IOP Conference Publication), vol. 147, 2016.

[10] R. Kumar and M. Kumar, 'Size dependence of thermoelastic properties of nanomaterials", International Journal of Nanoscience, 9(5), pp. 537542, 2010.

[11] G. Sharma, S. Bhatt, R. Kumar and M. Kumar, "Size, Shape and Temperature Effect on Nanomaterials", Indian Journal of Pure and Applied Physics, vol. 53, pp. 768-775, 2015.

[12] W. H. Qi and M. P. Wang, "Size effect on the cohesive energy of nanoparticle", Journal of Materials Science Letters, v. 21, pp. 1743-1745, 2002.

[13] Y. D. Qu, X. L. Liang, X. Q. Kong and W. Z. Zhang, "Size dependent cohesive energy, melting temperature and debye temperature of spherical metallic nanoparticles", Physics of metals and metallography, $\mathrm{v}$. 118(6), pp. 528534, 2017.

[14] R. Kumar and M. Kumar, "Effect of size on the cohesive energy, melting temperature and debye temperature of nanomaterials", Indian Journal Of Pure and Applied Physics, v. 50, pp. 329-334, 2012.

[15] R. Kumar, G. Sharma, M. Kumar, "Size and Temperature Effect on Thermal Expansion Coefficient and Lattice Parameter of Nanomaterials", Modern Physics Letters B (World Scientific Publishing Company), vol. 27, 2013.

[16] G. Guisbiers, "Size and shape dependencies of nanomaterial properties: Thermodynamic considerations", Materials Research Society Symposium Proceedings, v. 1371, pp. 1-6, 2012.

[17] M. Manu and V. Dubey, "Theoretical modeling on thermal expansion of $\mathrm{Al}$. Ag and $\mathrm{Cu}$ nanomaterials", American Institute of Physics Conference Proceedings., v. 1953(030191), pp. 1-5. 
[18] M. Zhao and Q. Jiang, "Size effect on thermal properties in low-dimensional materials", Key Engineering Materials, v. 444, 189-217, 2010.

[19] Y. F. Zhu, J. S. Lian and Q. Jiang, "Modeling of the melting point, debye temperature, thermal expansion coefficient and the specific heat of nanostructured materials", The Journal of Physical Chemistry C., v. 113, pp. 16896-16900, 2009

[20] C. Kittel, Introduction to solid state physics, $8^{\text {th }}$ Ed., John Wiley and Sons, Inc., USA, pp. 20, 2004.

[21] R. Kumar, G. Sharma and M. Kumar, "Effect of size and shape on the vibrational and thermodynamic properties of nanomaterials", Journal of Thermodynamics, pp. 1-5, 2013.

[22] C. C. Yang, M. X. Xiao, W. Li and Q. Jiang, "Size effects on the debye temperature, Einstein temperature and volume thermal expansion coefficient of nanocrystals", Solid State Communication, v. 139, pp. 148-152, 2006

[23] L. Lihong, Li Meizhi, Q. Fuqi and W. Yueguang, "Temperature Effect on Elastic Modulus of Thin Films and Nanocrystals", Philosophical Magazine, vol. 93, pp. 574-583, 2013.

[24] X. Y. Zhou, B. L. Huangb and T. Y. Zhang, "Size and Temperature Dependent Young's Modulus and Size Dependent Thermal Expansion Coefficient of Thin Films", Royal Society of Chemistry, vol. 18, 2016.

[25] Shridhar Pathak, BK Pandey, Ratan L Jaiswal, "Modelling for the Study of thermoelastic Properties of Nanoparticles" Adv. Mater. Lett., 12(2): 21021605, 2021 